\title{
ANALYTIC CAPACITY, CALDERÓN-ZYGMUND OPERATORS, AND RECTIFIABILITY
}

\author{
GUY DAVID
}

\begin{abstract}
For $K \subset \mathbb{C}$ compact, we say that $K$ has vanishing analytic capacity (or $\gamma(K)=0$ ) when all bounded analytic functions on $\mathbb{C} \backslash K$ are constant. We would like to characterize $\gamma(K)=0$ geometrically. Easily, $\gamma(K)>0$ when $K$ has Hausdorff dimension larger than 1 , and $\gamma(K)=0$ when $\operatorname{dim}(K)<1$. Thus only the case when $\operatorname{dim}(K)=1$ is interesting. So far there is no characterization of $\gamma(K)=0$ in general, but the special case when the Hausdorff measure $H^{1}(K)$ is finite was recently settled. In this case, $\gamma(K)=0$ if and only if $K$ is unrectifiable (or Besicovitchirregular), i.e., if $H^{1}(K \cap \Gamma)=0$ for all $C^{1}$-curves $\Gamma$, as was conjectured by Vitushkin.

In the present text, we try to explain the structure of the proof of this result, and present the necessary techniques. These include the introduction to Menger curvature in this context (by M. Melnikov and co-authors), and the important use of geometric measure theory (results on quantitative rectifiability), but we insist most on the role of Calderón-Zygmund operators and $T(b)$ Theorems.
\end{abstract}

\section{Introduction}

The aim of this text is to give an account of developments in CalderónZygmund theory and geometric measure theory (rectifiability) that led to a proof of Vitushkin's conjecture on analytic capacity. These developments will be presented with a definite bias; a big part of the agenda for this text is to convince the reader that this problem about analytic capacity needed a fair amount of technology to be solved, and thus it was natural that it waited essentially until now to be solved, and that it was likely that this would happen about now. 
We shall insist here a little more on the aspects related to singular integral operators, and leave the geometric measure theory more in the shadow. This is definitely not by lack of interest, but rather to make the exposition easier.

For a more impartial account, the reader may consult [Ma5] and its references.

The author wishes to dedicate this work to the memory of A. P. Calderón. This is a little presomptuous, but certainly not out of context, since the main result in the theory of analytic capacity is probably Calderón's contribution to the proof of the Denjoy conjecture. (See the beginning of Section 4.)

It is a pleasure to thank Joan Verdera for a careful reading of this text and various improvements.

\section{Analytic capacity}

Let $K$ be a compact subset of the complex plane. The main question for these notes is "When are there nonconstant bounded analytic functions on $\mathbb{C} \backslash K$ ?" This is known as Painlevé's problem, and it has only been partially solved so far (see Theorem 3.4 below).

In 1945, L. Ahlfors [Ah] introduced the following set function $\gamma$ (analytic capacity) that measures how many bounded analytic functions on $\mathbb{C} \backslash K$ there are. Denote by $H^{\infty}(\mathbb{C} \backslash K)$ the set of bounded analytic functions on $\mathbb{C} \backslash K$. First note that if $f \in H^{\infty}(\mathbb{C} \backslash K)$, it has a removable singularity at infinity; this allows us to define $f(\infty)=\lim _{z \rightarrow \infty} f(z)$ and $f^{\prime}(\infty)=\lim _{z \rightarrow \infty} z(f(z)-f(\infty))$. Now set

$$
\gamma(K)=\sup \left\{f^{\prime}(\infty) ; f \in H^{\infty}(\mathbb{C} \backslash K), f(\infty)=0\right\} .
$$

It is easy to see that there are nonconstant functions in $H^{\infty}(\mathbb{C} \backslash K)$ if and only if $\gamma(K)>0$. The main result in $[\mathbf{A h}]$ is that $\gamma(K)=0$ if and only if $K$ is "removable for bounded analytic functions", that is, if for all open sets $\mathcal{U} \supset K$ and all bounded analytic functions $f$ on $\mathcal{U} \backslash K, f$ has a (bounded holomorphic) extension to $\mathcal{U}$. Note that $\gamma(K)=0$ in (2.1) corresponds to the case when $\mathcal{U}=\mathbb{C}$ (where we can use Liouville's theorem to say that $H^{\infty}(\mathbb{C})$ is trivial), and Ahlfors says that removability in $\mathbb{C}$ implies removability in all open neighborhoods of $K$.

The question that Ahlfors does not really address, and which is at the center of this text, is the geometric characterization of vanishing analytic capacity. Before we get to the conjecture of Vitushkin, let us record some of the elementary properties of $\gamma$. 
2.2. If $K$ is thick enough, i.e., if its Hausdorff $\operatorname{dimension} \operatorname{dim} K$ is strictly more than 1 , then $\gamma(K)>0$. Indeed, Frostman's lemma says that there is a nontrivial positive measure $\mu$ on $K$ such that $\mu(B(x, r)) \leq C r^{1+\epsilon}$ for some positive constants $\epsilon$ and $C$ (and all disks $B(x, r))$. It is then easy to check that the convolution $f=\frac{1}{z} * \mu$ lies in $H^{\infty}(\mathbb{C} \backslash K)$, with $f^{\prime}(\infty)=\mu(K)>0$.

2.3. If $K$ is too small, i.e., if $\operatorname{dim} K<1$ or even $\operatorname{dim} K=1$ but $H^{1}(K)=0$, then $\gamma(K)=0$. Recall that $H^{1}(K)$, the one-dimensional Hausdorff measure of $K$, is the natural generalization of "the total length of $K$ " when $K$ is not smooth enough. See [Fal] or [Ma2] for definitions. This observation is due to Painlevé. The point is that if $f \in H^{\infty}(\mathbb{C} \backslash K)$, $f(\infty)=0$, and $z \in \mathbb{C} \backslash K$, then for each $\epsilon>0$ one can surround $K$ by a finite collection of curves $\Gamma_{i}$, with $\sum_{i} \operatorname{length}\left(\Gamma_{i}\right)<\epsilon$ and $z$ outside of the $\Gamma_{i}$. For $\epsilon$ small enough, $\operatorname{dist}\left(\Gamma_{i}, z\right) \geq \frac{1}{2} \operatorname{dist}(z, K)$, and Cauchy's formula yields

$$
\begin{aligned}
|f(z)|=\left|\frac{1}{2 \pi i} \sum_{i} \int_{\Gamma_{i}} \frac{f(w) d w}{z-w}\right| \\
\quad \leq \frac{1}{\pi}\|f\|_{\infty} \operatorname{dist}(z, K)^{-1} \sum_{i} \operatorname{length}\left(\Gamma_{i}\right) \leq C \epsilon,
\end{aligned}
$$

so that $f(z)=0$.

2.5. If $K$ is connected and not reduced to a point, then $\gamma(K)>0$. One can take for $f$ (the restriction to $\mathbb{C} \backslash K$ of) a conformal mapping from the complement of $K$ in the Riemann sphere to the unit disk. A slightly more careful estimate using the Koebe $\frac{1}{4}$-theorem even gives that $\gamma(K) \geq \frac{1}{4} \operatorname{diam} K$. This example is more interesting than it seems, because apparently this is the only case where we can get positive analytic capacity without obtaining the corresponding bounded analytic functions as the Cauchy integrals of some measures.

This completes our short list of simple properties of $\gamma$. For more information on analytic capacity, we refer the reader to $[\mathbf{G a 2}],[\mathbf{M a 2}]$, [Ve1], or [Vi2].

In this text we take it for granted that analytic capacity and the Painlevé problem are very interesting. Some justification for this, and a description of the relations with the theory of approximation, can be found in $[\mathbf{V e 1}]$ and $[\mathbf{V i 2}]$. 


\section{Vitushkin's conjecture}

We now focus on the Painlevé problem. Because of 2.2 and 2.3 we may restrict to compact sets $K$ with $\operatorname{dim} K=1$ and $H^{1}(K)>0$.

When $K$ is a smooth enough curve $\left(C^{1+\epsilon}\right.$ is enough), the Cauchy integral of a bump function on $K$ is bounded, and thus $\gamma(K)>0$. It is also not too difficult to show that $\gamma(K)>0$ when $K$ is a subset with positive measure of such a curve.

On the other hand, as early as 1959, A. G. Vitushkin [Vi1] gave an example of a compact set $K$ such that $H^{1}(K)>0$ but $\gamma(K)=0$. Thus size is not the only thing that matters.

Vituskin's example was quite complicated, but eventually J. Garnett [Ga1] and L. D. Ivanov [Iv] found a much simpler one: the Cantor set of dimension 1 (and even such that $H^{1}(K)=\sqrt{2}$ ) constructed as follows. Start from the unit square $Q_{0}$; replace it with the union of the 4 squares of sidelength $\frac{1}{4}$ contained in $Q_{0}$ and situated in its corners; next replace each square with 4 squares of sidelength $4^{-2}$ situated in its corners; iterate, and finally take the intersection.

Even before that, Vitushkin [Vi2] conjectured that $\gamma(K)=0$ if and only if

$$
H^{1}\left(\pi_{\theta}(K)\right)=0 \text { for almost every direction } \theta
$$

where $\pi_{\theta}$ denotes the orthogonal projection from the plane to the line $\mathcal{L}_{\theta}=\{x+i y: x \cos \theta+y \sin \theta=0\}$, say. In the special case when

$$
0<H^{1}(K)<+\infty
$$

(or even when $H^{1}(K)$ is sigma-finite), (3.1) is one of the many characterizations of "unrectifiability" (also called "irregularity" by Besicovitch). The most common definition of unrectifiability is that

$$
H^{1}(K \cap \Gamma)=0 \text { for all } C^{1} \text {-curves } \Gamma \text {, }
$$

but one also gets an equivalent definition by replacing $C^{1}$-curves with rectifiable curves (i.e., curves with finite length) or graphs of Lipschitz functions (possibly rotated).

In the special case of (3.2), Vitushkin was right, and the proof of the following theorem was recently completed.

Theorem 3.4. Let $K$ be a compact set in the plane such that $0<H^{1}(K)<+\infty$. Then $\gamma(K)=0$ if and only if $K$ is unrectifiable, i.e., if and only if (3.3) holds. 
Thus Theorem 3.4 solves the Painlevé problem when $H^{1}(K)<+\infty$. Before we discuss its proof, let us say a few words about the (difficult) case when $H^{1}(K)=+\infty$ (but $\operatorname{dim} K=1$ ), which remains unsolved. In this case all the usual characterizations of unrectifiability (when (3.2) holds) are no longer equivalent, and actually none of them seems to characterize $\gamma(K)=0$. [See the last section for a conjecture of Melnikov, though.]

The fact that (3.1) does not characterize $\gamma(K)=0$ in general was first proved by P. Mattila [Ma1]. His proof had the amusing feature that it did not say which of the two implications was false: he just proved that $\gamma(K)=0$ is preserved by conformal mappings and that (3.1) is not. Later P. Jones and T. Murai [JoMu] gave examples of sets $K$ such that $\gamma(K)>0$ but $(3.1)$ holds. The converse is not known to be true or false yet.

The situation where $H^{1}(K)=+\infty$ is still far from clear. For most of the rest of this text, we shall restrict to the case when $H^{1}(K)<+\infty$.

\section{The Cauchy integral}

It is not too surprising that analytic capacity is closely related to properties of the Cauchy kernel. In particular, the main (if not most recent!) part of Theorem 3.4, i.e., the fact that

$$
\gamma(K)>0 \text { if } H^{1}(K \cap \Gamma)>0 \text { for some } C^{1} \text {-curve } \Gamma,
$$

is a consequence of A. P. Calderón's result $[\mathbf{C a}]$ on the $L^{2}$-boundedness of the Cauchy operator on (small) Lipschitz graphs.

For any (finite) positive measure $\mu$ without atoms and $f \in L^{2}(d \mu)$, define $\mathcal{C}_{\mu} f$ by

$$
\mathcal{C}_{\mu} f(z)=\int \frac{f(w) d \mu(w)}{z-w}
$$

This is well defined for $z \in \mathbb{C} \backslash \operatorname{supp} \mu$; in the present text we shall do as if it was also well defined on the support of $\mu$. Normally we should be much more careful, consider truncated integrals, and then take weak limits or principal values after showing uniform estimates. These issues are not central here, and so we shall not bother.

If $\Gamma \subset \mathbb{C}$ is a rectifiable curve, we shall set $\mathcal{C}^{\Gamma}=\mathcal{C}_{\mu}$, where $\mu$ denotes the restriction of $H^{1}$ to $\Gamma$ (i.e., $\mu$ is the arclength measure on $\Gamma$ ). The boundedness of $\mathcal{C}_{\Gamma}$ on $L^{2}(\Gamma, d \mu)$ when $\Gamma$ is a Lipschitz graph was a very important issue in analysis for quite a few years, independently of any 
connection with analytic capacity. For analytic capacity, the most relevant theorem is due to Calderón $[\mathbf{C a}]$, and says that there is a very small constant $\epsilon_{0}>0$ such that

(4.3) $\mathcal{C}^{\Gamma}$ is a bounded operator on $L^{2}(\Gamma, d \mu)$ when $\Gamma=\{x+i y ; x \in \mathbb{R}$ and $y=A(x)\}$ is the graph of a Lipschitz function $A: \mathbb{R} \rightarrow \mathbb{R}$, with $|A(x)-A(y)| \leq \epsilon_{0}|x-y|$.

Let us, incidentally, remind the reader that the study of $\mathcal{C}^{\Gamma}$ culminated with the theorem of Coifman, McIntosh and Meyer $[\mathbf{C M M}]$, who proved that (4.3) holds for all $\epsilon_{0}>0$. Analysts were so interested in the result that they continued giving new proofs, essentially up until now, and often with good reasons.

The question of $L^{2}$-boundedness of $\mathcal{C}^{\Gamma}$ for rectifiable curves was settled a little later [Da1] with the following slight improvement of [CMM]:

(4.4) For a rectifiable curve $\Gamma, \mathcal{C}^{\Gamma}$ is bounded on $L^{2}(\Gamma, d \mu)$ if and only if $\Gamma$ is an Ahlfors-regular curve.

Recall that (Ahlfors-)regular curves are rectifiable curves $\Gamma$ such that

$$
H^{1}(\Gamma \cap B(x, r)) \leq C r \text { for all } x \in \mathbb{C} \text { and } r>0,
$$

and some constant $C$. The reader should not worry about the precise definition of a curve or how it is parameterized; regular curves are almost the same as connected sets that satisfy (4.5), and the difference will certainly not matter here.

We shall see in Section 11 that we even know now for which measures $\mu$ the Cauchy operator $\mathcal{C}_{\mu}$ is bounded on $L^{2}(d \mu)$.

To conclude this section, let us rapidly indicate how (4.1) can be deduced from (4.3). Let $K$ be such that $H^{1}(K \cap \Gamma)>0$ for some $C^{1}$-curve $\Gamma$. By (4.3) (and maybe after cutting $\Gamma$ into a finite number of pieces), $\mathcal{C}^{\Gamma}$ is bounded on $L^{2}\left(\Gamma, d \mu\right.$ ) (where $d \mu$ still denotes the restriction of $H^{1}$ to $\Gamma)$. If we also knew that $\mathcal{C}^{\Gamma}$ maps $L^{\infty}(\Gamma, d \mu)$ to itself, then we would get that $f=\mathcal{C}^{\Gamma}\left(\mathbb{1}_{K \cap \Gamma}\right)$ is bounded and holomorphic away from $K$, by the maximum principle and modulo issues of convergence and truncature that we decided to ignore here. Of course $\mathcal{C}^{\Gamma}$ is not bounded on $L^{\infty}(\Gamma)$, but standard Calderón-Zygmund theory says that this only barely fails (in fact, $\mathcal{C}^{\Gamma}$ sends $L^{\infty}(\Gamma)$ to some BMO space). In this situation, it is still possible to use duality, the fact that $\mathcal{C}^{\Gamma}$ maps $L^{1}(\Gamma)$ to weak- $L^{1}(\Gamma)$ (again, by standard Calderón-Zygmund theory) and Hahn-Banach to prove that there are bounded functions $h$ supported on $K \cap \Gamma$ such that $\mathcal{C}^{\Gamma}(h) \in H^{\infty}(\mathbb{C} \backslash K)$. See for instance [Ch1] for details about this proof. 
Apparently, the fact that the $L^{2}$-boundedness of the Cauchy operator implies (4.1) was known from Havin and Havinson [HaHa] before the proof of (4.3); when Calderón proved (4.3), he did not know about potential applications to (4.1), and the argument was rediscovered by D. Marshall (who later found out about [HaHa]). The duality argument alluded to above is due to Uy $[\mathbf{U y} \mathbf{1}]$, and was later rediscovered by Davie and Øksendal [DØK].

\section{Calderón-Zygmund operators and $T(b)$}

This section looks a lot like a digression from our main topic, but we shall need to refer to it later. We start with a few definitions, which will be given on $\mathbb{R}^{n}$ for simplicity.

A "standard kernel" is a continuous function $K:\left(\mathbb{R}^{n} \times \mathbb{R}^{n}\right) \backslash \Delta \rightarrow \mathbb{C}$, where $\Delta=\left\{(x, x) ; x \in \mathbb{R}^{n}\right\}$ is the diagonal, and that satisfies the following size and mild regularity conditions:

$$
|K(x, y)| \leq C|x-y|^{-n}
$$

and

$$
\left|K(x, y)-K\left(x^{\prime}, y\right)\right|+\left|K(y, x)-K\left(y, x^{\prime}\right)\right| \leq C \frac{\left|x^{\prime}-x\right|^{\delta}}{|x-y|^{n+\delta}}
$$

for all $x, x^{\prime}, y \in \mathbb{R}^{n}$ such that $\left|x^{\prime}-x\right|<\frac{1}{2}|x-y|$ (say), and where $0<\delta \leq 1$ and $C \geq 0$ are two constants. [Other, slightly weaker conditions are possible, but this will be more than general enough.]

The most convenient way to define singular integral operators is in a weak form, by viewing $T$ as a bilinear from and defining $\langle T f, g\rangle$ (the effect of $T f$ on the test function $g$ ) for $f, g$ in a dense class $\mathcal{D}$. Most of the time, we take $\mathcal{D}=\mathcal{C}_{c}^{\infty}\left(\mathbb{R}^{n}\right)$, but in some cases (like for Theorem 5.6 below), other choices are more convenient.

We say that $T$ is a singular integral operator if there is a standard kernel $K$ as above such that

$$
\langle T f, g\rangle=\iint K(x, y) f(y) g(x) d y d x
$$

for all $f, g \in \mathcal{D}$ such that $\operatorname{support}(f)$ is disjoint from $\operatorname{support}(g)$. This definition may seem complicated, but it is a nice way of implying that (morally)

$$
T f(x)=\int K(x, y) f(y) d y
$$


without having to worry (yet) about issues of convergence or truncatures in (5.4). This has the slight inconvenient that $K$ does not determine $T$. A typical difference between two singular integral operators with the same kernel is an operator of pointwise multiplication by some function.

When the standard kernel $K$ is antisymmetric, i.e., when $K(y, x)=$ $-K(x, y)$ for all $x, y$, there is a natural way to define a singular integral operator $T$ using $K$ : set

$$
\langle T f, g\rangle=\lim _{\epsilon \rightarrow 0}\left\{\iint_{|x-y|>\epsilon} K(x, y) f(y) g(x) d y d x\right\} .
$$

The limit is fairly easily seen to exist, because of (5.1) and the antisymmetry. We'll refer to the operator $T$ defined by (5.5) as the principal value operator defined by $K$.

A very typical example of $T$ and $K$ comes from the operator $\mathcal{C}^{\Gamma}$ presented above, when $\Gamma$ is the graph of the Lipschitz function $A$ (as in (4.3)). If we use the parameterization $x \rightarrow x+i A(x)$ of $\Gamma$ to reduce to an operator acting on $\mathbb{R}$, we see that $\mathcal{C}^{\Gamma}$ is equivalent to the principal value operator $T_{A}$ defined by the standard, antisymmetric kernel

$$
K(x, y)=[x+i A(x)-y-i A(y)]^{-1} .
$$

For a long time, what was best understood (and made the early reputation of Calderón-Zygmund operators) was the various boundedness properties of $T$ (boundedness on $L^{p}, 1<p<+\infty$, of $T$ and the maximal operator $T^{*}$; boundedness from $L^{1}$ to weak- $L^{1}$ and from $L^{\infty}$ to BMO) as soon as we know that $T$ extends to a bounded operator on $L^{2}\left(\mathbb{R}^{n}\right)$. Here BMO is the space of functions with bounded mean oscillation (a space a little larger than $L^{\infty}$ ). For nonconvolution operators, obtaining $L^{2}$-boundedness was often a problem, as in the case of $\mathcal{C}^{\Gamma}$ and $T_{A}$ above. The following result helps.

Theorem 5.6 [DJS]. Let $b$ be a bounded, complex-valued function on $\mathbb{R}^{n}$. Suppose that b is "accretive", i.e., that

$$
\Re \text { e } b(x) \geq \delta \text { for all } x \in \mathbb{R}^{n}
$$

and some constant $\delta>0$. Let $T$ be a singular integral operator (as above). Then $T$ extends to a bounded operator on $L^{2}\left(\mathbb{R}^{n}\right)$ if and only if

$$
T b \in \mathrm{BMO}, T^{t} b \in \mathrm{BMO} \text {, and } T \text { is weakly bounded. }
$$


This statement is rather vague; for a precise one, as well as for information on Calderón-Zygmund operators, the reader may consult [CoMe], [Mey], [Da3] or [St] for instance. Let us just give a few comments. In the definition of singular integral operators above, it is easiest to take $\mathcal{D}=b \mathcal{C}_{c}^{\infty}\left(\mathbb{R}^{n}\right)$. The definition of $T b$ (modulo an additive constant) is fairly easy (by duality). Here $T^{t}$ denotes the transpose of $T$, defined by $\left\langle T^{t} f, g\right\rangle=\langle T g, f\rangle$; it is also a singular integral operator. The weak boundedness is an additional technical condition related to scale invariance, and which is in principle easy to check. It typically rules out operators like differential operators.

When $T$ is the principal value operator defined by a standard antisymmetric kernel, then $T^{t}=-T$ and the weak boundedness is automatic, and so (5.8) may be replaced with the only condition that $T b \in \mathrm{BMO}$.

The accretivity condition on $b$ is natural; without it, the cancellation hidden in the requirement that $T b \in$ BMO could come from $b$ itself, rather than $T$. It can be weakened somewhat, though. For instance, it is enough that

$$
\left|\int_{Q} b(x) d x\right| \geq \delta|Q| \text { for all dyadic cubes } Q .
$$

In the case of the Cauchy operator $T_{A}$ above, and when $A$ is Lipschitz, Theorem 5.6 is easy to apply: we take $b(x)=1+i A^{\prime}(x)$, and Cauchy's formula says that $T_{A} b=0$. Hence $T_{A}$ is bounded (as was known from $[\mathrm{CMM}])$.

Of course these developments of Calderón-Zygmund theory were not done with analytic capacity in mind, and at this point of the exposition it may seem that the only connections with Painlevé's problem are through (4.3) and (4.1). We shall see later that, amusingly, a version of Theorem 5.6 seems to be required for the proof of the converse.

\section{Spaces of homogeneous type}

Let us continue our digression with comments on spaces of homogeneous type and doubling measures. Let us not give the most general definition here, but only a fairly general special case. Our spaces of homogeneous type will be metric spaces ( $E$, dist) with a (nonatomic) locally finite measure $\mu$ supported on $E$, and which satisfy the "doubling property"

$$
\mu(B(x, 2 r) \leq C \mu(B(x, r)) \text { for all } x \in E \text { and } r>0,
$$

and some constant $C \geq 1$. 
The reader is welcome to think of $E$ as a subset of some $\mathbb{R}^{N}$, with the usual Euclidean distance. An interesting special case of this is the case of Ahlfors-regular sets of dimension $d$ (in $\mathbb{R}^{N}$ ), i.e., closed sets of locally finite $d$-dimensional Hausdorff measure such that

$$
\begin{aligned}
C^{-1} r^{d} \leq H^{d}(E \cap B(x, r)) & \leq C r^{d} \\
& \text { for } x \in E \text { and } 0<r<\operatorname{diameter}(E) .
\end{aligned}
$$

It has been common knowledge among specialists that spaces of homogeneous type are the right setup for most of Calderón-Zygmund theory. See for instance [CoWe], [MaSe1] and [MaSe2]. In such a setting, one replaces (5.1) with

$$
|K(x, y)| \leq C \mu(B(x, \operatorname{dist}(x, y)))^{-1}
$$

and (5.2) with an appropriate extra smoothness assumption, like

$$
\begin{aligned}
\left|K(x, y)-K\left(x^{\prime}, y\right)\right| & +\left|K(y, x)-K\left(y, x^{\prime}\right)\right| \\
& \leq C\left\{\frac{\operatorname{dist}\left(x, x^{\prime}\right)}{\operatorname{dist}(x, y)}\right\}^{\delta} \mu(B(x, \operatorname{dist}(x, y)))^{-1} .
\end{aligned}
$$

Note, by the way, that if $(E$, dist, $\mu)$ is a space of homogeneous type and $a>0$ is any power, then $\left(E, \operatorname{dist}^{a}, \mu\right)$ is also a space of homogeneous type. With the definition above, we should restrict to powers $a$ such that dist $^{a}$ is also a distance, but even this is not a serious issue (the precise triangle inequality with constant 1 is not an absolute necessity in the definitions). When we replace dist with $\operatorname{dist}^{a}$, we do not alter (6.3) or (6.4) seriously, which is of course a good sign.

Essentially all the standard results about singular integral operators and boundedness extend to spaces of homogeneous type, often with very similar proofs. Usually, the extension is difficult to find in the literature (precisely because the proof is the same, but longer). In the case of Theorem 5.6, the first proof of extension was due to R. Coifman (unpublished) and was rather painful, but fortunately the proof of Theorem 5.6 (when $b=1$ ) given in [CJS] (using the matrix of $T$ in the Haar system) extends nicely. [See for instance [Da3] for a little more detail.] The main useful point for this is the existence on $(E$, dist, $\mu)$ of analogues of the usual dyadic cubes in $\mathbb{R}^{n}$, as in [Da2] (but rather consult the appendix in $[\mathrm{Da} 3]$ or $[\mathrm{Ch2}])$.

The consequence of all this is that in the context of Section 4, we may apply Theorem 5.6 to decide whether $\mathcal{C}_{\mu}$ is a bounded operator on $L^{2}(d \mu)$, provided that $\mu$ is a doubling measure (as in (6.1)). 


\section{Menger curvature}

The Menger curvature of a triple $(x, y, z)$ of points in the plane is the inverse of the radius of the circle through $x, y, z$. It will be denoted by $c(x, y, z)$. When $x, y, z$ lie on a same line, we set $c(x, y, z)=0$. These numbers were introduced in the context of analytic capacity by M. Melnikov [Mel], and are very important because of the following magic formula: for $z_{1}, z_{2}, z_{3} \in \mathbb{C}$,

$$
\sum_{\sigma \in S_{3}} \frac{1}{z_{\sigma(1)}-z_{\sigma(2)}} \frac{1}{\overline{z_{\sigma(1)}-z_{\sigma(3)}}}=c\left(z_{1}, z_{2}, z_{3}\right)^{2},
$$

where we sum over the set $S_{3}$ of permutations of $\{1,2,3\}$. The magic comes from the fact that $c(x, y, z) \geq 0$, as we shall see in the following computation taken from $[\mathbf{M e V e}]$.

Let $\mu$ be a finite positive measure on the plane, without atoms, and suppose for security reasons that $\mu$ has "linear growth", i.e., that

$$
\mu(B(x, r)) \leq C r \text { for all } x \in \mathbb{C} \text { and } r>0 .
$$

Then

$$
\left\|\mathcal{C}_{\mu}(1)\right\|_{L^{2}(d \mu)}^{2}=\int_{z_{1}}\left\{\int_{z_{2}} \frac{d \mu\left(z_{2}\right)}{z_{1}-z_{2}}\right\}\left\{\int_{z_{3}} \frac{d \mu\left(z_{3}\right)}{z_{1}-z_{3}}\right\}^{-} d \mu\left(z_{1}\right) .
$$

Here and in the computations that follow, we should normally be more careful, truncate integrals, prove uniform estimates, and go to the limit. With our security assumption (7.2), it turns out that the computations presented here in a careless way can be justified without too much trouble.

The right-hand side of (7.3) is a triple integral; we do not modify it if we symmetrize it (as in (7.1)), and hence (7.1) gives

$$
\left\|\mathcal{C}_{\mu}(1)\right\|_{2}^{2}=\frac{1}{6} \iiint c\left(z_{1}, z_{2}, z_{3}\right)^{2} d \mu\left(z_{1}\right) d \mu\left(z_{2}\right) d \mu\left(z_{3}\right),
$$

modulo errors of finite size that come from truncatures.

This is very good to know, because for the first time it gives a direct connection between boundedness properties of $\mathcal{C}_{\mu}$ and a right-hand side with a definite geometric meaning.

In [MeVe], M. Melnikov and J. Verdera used (7.4) and localized versions of it (that is, where 1 is replaced with characteristic functions of disks) to give a new short proof of boundedness of $\mathcal{C}^{\Gamma}$ when $\Gamma$ is a Lipschitz graph. The argument just needs Theorem 5.6 with $b \equiv 1$ and a direct estimate on curvature (as in the right-hand side of (7.4)) on the graph.

The most striking application of (7.4) was the converse result in [MMV]. 
Theorem 7.5 [MMV]. If $E$ is an Ahlfors-regular set of dimension 1 in the plane and $\mu$ denotes the restriction of $H^{1}$ to $E$, then $\mathcal{C}_{\mu}$ has a bounded extension to $L^{2}(E, d \mu)$ if and only if $E$ is uniformly rectifiable, i.e., if $E \subset \Gamma$ for some Ahlfors-regular curve $\Gamma$.

See (6.2) for Ahlfors-regularity, and (4.4) and (4.5) for the direct part of Theorem 7.5 and Ahlfors-regular curves. For the converse, one starts with the observation that if $\mathcal{C}_{\mu}$ is bounded on $L^{2}(d \mu)$, then (7.4) (applied to $\left.\mathbb{1}_{B(x, r)} d \mu\right)$ gives

$$
\iiint_{B(x, r)^{3}} c\left(z_{1}, z_{2}, z_{3}\right)^{2} d \mu d \mu d \mu \leq C r .
$$

The rest of the proof is purely geometric: for Ahlfors-regular sets $E$, (7.6) happens to imply uniform rectifiability. There is still no very simple proof of this, but on the other hand the geometric measure theory needed for the proof was already available at the time of [MMV].

We shall rapidly return in Section 11 to the issue of $L^{2}$-boundedness of $\mathcal{C}_{\mu}$ when $\mu$ is not assumed to be Ahlfors-regular (or doubling).

\section{Some geometric measure theory}

We have just seen how Menger curvature allows us to translate properties of the Cauchy operator (which themselves are connected to analytic capacity) into geometric properties. To complete the proofs, it is good to have ways to connect various geometric quantities (like the curvature integrals in (7.4) and (7.6)) to other ones, and eventually to simpler rectifiability properties.

Maybe it is worth pointing out that the tools of geometric measure theory described in this section are one of the most important ingredients of the proof, and also the part that I like best. We shall say very little about them here because we decided to take a point of view that would be more oriented in the direction of Calderón-Zygmund theory. See [Da4] for a short survey on the results of this section.

Recall that a set $E$ with sigma-finite $H^{1}$-measure is said to be rectifiable if it is contained in a countable union of $C^{1}$-curves, plus perhaps a set of vanishing $H^{1}$-measure. As with the definition of the opposite notion unrectifiability (see near $(3.3)$ ), $C^{1}$ curves may be replaced with curves of finite length without changing the notion. It is a fairly direct consequence of definitions that any set with sigma-finite measure is the disjoint union of a rectifiable piece and an unrectifiable piece, in an essentially unique way. 
There is a very beautiful classical theory of rectifiability, where rectifiable sets are shown to have lots of good properties, and unrectifiable sets to have the corresponding "bad" properties (existence of weak tangents or density almost nowhere, (3.1), etc...). See for instance [Ma2] (or standard references, like $[\mathbf{F e}]$ or $[\mathbf{F a l}])$ for more information.

It took most of us a relatively long time to understand that the $L^{2}$ boundedness of singular integral operators like $\mathcal{C}^{E}$ on sets $E$ should be related to rectifiability properties of $E$. Then one had to find ways to quantify rectifiability (because the boundedness of an operator, unlike (standard) rectifiability, is a very quantitative notion; there is even a norm to measure it). We shall just give a few examples of this; a very systematic description can be found in the first part of [DaSe].

A typical example of numbers that measure how rectifiable a set $E$ is are the $\mathbf{P}$. Jones numbers $\beta(x, t)$. These were introduced in $[\mathbf{J o 1}]$ in the context of the Cauchy integral operators; they are defined by

$$
\beta(x, t)=\inf _{P}\left\{\frac{1}{t} \sup _{y \in E \cap B(x, t)} \operatorname{dist}(y, P)\right\},
$$

where the infimum is taken over all choices of lines $P$ (and with the convention that $\beta(x, t)=0$ when $E \cap B(x, t)=\emptyset)$. Obviously $\beta(x, t)$ is a scale-invariant measurement of how close $E \cap B(x, t)$ stays to a line. Here is a quantitative rectifiability result.

Theorem 8.2 [Jo2]. Let $E$ be a compact subset of the plane. Then there is a curve $\Gamma$ with finite length that contains $E$ if and only if

$$
\iint_{\mathbb{R}^{2}} \int_{0}^{\operatorname{diam} K} \beta(x, t)^{2} \frac{d x d t}{t^{2}}<+\infty .
$$

The proof also gives that the smallest length of $\Gamma, \Gamma \supset E$, is equivalent to diam $K+\beta_{E}^{2}$, where $\beta_{E}^{2}$ is the left-hand side of (8.3). Note that this can be seen as a traveling salesman problem with an infinite set $E$. A slightly simpler variant of Theorem 8.2 is the following uniform version with regular sets.

Theorem 8.4 [Jo2]. Let $E$ be a one-dimensional Ahlfors-regular set. Then $E$ is uniformly rectifiable if and only if there is a constant $C$ such that

$$
\int_{x \in E \cap B(a, r)} \int_{0}^{r} \beta(x, t)^{2} \frac{d H^{1}(x) d t}{t} \leq C r
$$

for all $a \in E$ and $r>0$. 
The different power of $t$ in the denominator should not shock the reader: here we are integrating on the 1-dimensional set $E$ rather than $\mathbb{R}^{2}$.

One of the nice features of uniform rectifiability is that characterizations of the type above are fairly stable. For instance, we may replace the sup in (8.1) by any $L^{p}$-mean, $1 \leq p<+\infty$, and still get a characterization of uniform rectifiability (for regular sets) by (8.5). There are also lots of other characterizations of uniform rectifiability by different types of numbers, in dimensions 1 (like here) and higher. See [DaSe].

Return to curvature. The fact that, for Ahlfors-regular sets, (7.6) implies uniform rectifiability is a not too hard consequence of the version of Theorem 8.4 where $\beta(x, t)$ has been defined with an $L^{2}$-mean. On top of the proof of this in $[\mathbf{D a S e}]$ there are two slightly more direct proofs (none of which too pleasant, though). The first one $([\mathbf{P a 1}])$ is a modification of the construction in $[\mathbf{J o 2}]$ that accomodates the $L^{2}$ means; the other one is an unpublished (so far) argument of P. Jones that reduces to Theorem 8.4 itself.

The following result, unlike the previous one and the results in [DaSe], was proved with analytic capacity in mind, and in fact answers a question of M. Melnikov. The fact that we won't assume lower bounds (or doubling conditions) on the mass of $E$ is important.

Theorem 8.6 [Lé]. Let $E$ be a compact set in the plane, suppose that $0<H^{1}(E)<+\infty$, let $\mu$ denote the restriction of $H^{1}$ to $E$, and set

$$
c^{2}(\mu)=\iiint c\left(z_{1}, z_{2}, z_{3}\right)^{2} d \mu d \mu d \mu
$$

(the total Menger curvature of $\mu$ ). If $c^{2}(\mu)<+\infty$, then $E$ is rectifiable.

This is one of the main ingredients in our proof of Theorem 3.4 (see Section 4). The proof is very nice, but a little technical. For a more friendly description (without much in terms of proofs), see [Da4].

\section{The last part of the proof}

We now give a rapid description of the proof of the converse part in Theorem 3.4, i.e., the fact that

(9.1) if $H^{1}(K)<+\infty$ and $K$ is unrectifiable (as in (3.3)), then $\gamma(K)=0$ 
This was first proved in $[\mathbf{D a} 5]$, relying on $[\mathbf{D a M a}]$, but there is a (just a little) more recent proof by F. Nazarov, S. Treil, and S. Volberg [NTV2]. Both proofs use the same sort of general scheme, but there are clever simplifying tricks in [NTV2] that make for a more pleasant and flexible proof.

To prove (9.1), we assume that $\gamma(K)>0$ and want to find some rectifiable piece in $K$. We are thus given a bounded holomorphic function $f$ on $\mathbb{C} \backslash K$, with $\|f\|_{\infty} \leq 1, f(\infty)=0$, and $f^{\prime}(\infty)=\gamma(K)>0$.

The first stage of the proof is to observe that there is a bounded measurable function $h$ on $K$ such that

$$
f=\mathcal{C}_{\mu}(h) \text { on } \mathbb{C} \backslash K,
$$

where we set $\mu=H_{\mid K}^{1}$ as usual. This is fairly easy to prove: essentially, we can surround $K$ by finite collections of curves with lengths $\leq C H^{1}(K)$, apply the Cauchy formula like in (2.4), and take an appropriate weak limit. See [Ma2, Theorem 19.9, p. 260] for a proof. Although this step looks quite benign, it strongly uses our assumption that $H^{1}(K)<+\infty$.

Suppose now that we were so lucky that we get $h \geq 0$. In this case the measure $h d \mu$ automatically satisfies the linear growth condition (7.2), because the measure of a disk for $h d \mu$ is a constant times the integral of $f$ on the corresponding circle (by an altogether easy integration by parts). Then, modulo a limiting argument that will be neglected here, $\mathcal{C}_{\mu}(h)$ is bounded, hence lies in $L^{2}(h d \mu)$, and then (7.4) (applied to $h d \mu$ ) gives that $c^{2}(h d \mu)<+\infty$ (with the notation of (8.7)).

Because $h$ is bounded and $\int h d \mu=f^{\prime}(\infty)>0$, we can find a closed subset $E$ of $K$ such that $H^{1}(E)>0$ and $h(x) \geq \delta>0$ on $E$. Then

$$
c^{2}\left(\mathbb{1}_{E} d \mu\right) \leq \delta^{-3} c^{2}(h d \mu)<+\infty .
$$

Theorem 8.6 now says that $E$ is rectifiable, as desired.

In general we have two difficulties to overcome. The first one is that $h d \mu$ may not have linear growth. This is not too hard to cope with; one can modify $h d \mu$ so that it has linear growth (essentially by removing pieces with too large a density and replacing them by little circles with a uniform measure on them). This can be done at the relatively low cost of removing a tiny bit of $K$, and getting a new function $\tilde{f}=\mathcal{C}_{\tilde{\mu}}(\tilde{h})$ with fairly good (BMO-like) integrability properties (instead of plain boundedness like for $f$ ). 
The serious difficulty comes from the fact that $h$ is complex-valued, not positive. It was addressed for the first time by M. Christ $[\mathbf{C h 2}]$ in the special case when $K$ is Ahlfors-regular. Here is a rapid description of the idea.

First suppose that $h$ is accretive (as in (5.7)). We can apply Theorem 5.6 on the space of homogeneous type $(K$, dist, $\mu$ ) (because $K$ is Ahlfors-regular). We take $b=h$, and note that $\mathcal{C}_{\mu}(b) \in L^{\infty} \subset$ $\operatorname{BMO}(K, d \mu)$. The other conditions in (5.8) are automatically satisfied by antisymmetry, and hence Theorem 5.6 gives the $L^{2}$-boundedness of $\mathcal{C}_{\mu}$. In particular, $\mathcal{C}_{\mu}(1) \in L^{2}(d \mu)$, and we can use (7.4) and conclude as above.

When $K$ is Ahlfors-regular but $h$ is not accretive, we have to be content with the only information that $\int h d \mu=f^{\prime}(\infty)>0$ to prevent cancellation. Then we want to replace $K$ and $h d \mu$ with a new regular set $\tilde{K}$ and a new measure $\tilde{h} d \tilde{\mu}$ on $\tilde{K}$ for which $\tilde{h}$ satisfies the weaker accretivity condition (5.9) (with $d \tilde{\mu}$ instead of $d x$, and where we use the analogue of dyadic cubes mentioned near the end of Section 6). The construction of $\tilde{K}$ and $\tilde{h} d \tilde{\mu}$ also uses these dyadic cubes, and a quite logical stopping time construction. Very roughly, one waits for the mean value of $h$ on cubes to become very small, and then one stops and replaces $K$ (in the corresponding cubes) with a tiny circle on which we make $\tilde{h}$ constant.

If the construction is done carefully, the mean value of $h$ on the cubes of $K$ that we replace with circles is very small. Thus the integral of $h d \mu$ on $K \backslash \tilde{K}$ (the union of those cubes) is very small. Since we know that $\int h d \mu=f^{\prime}(\infty)>0$, we get that most of $\int h d \mu$ comes from $K \backslash \tilde{K}$, and in particular $K \backslash \tilde{K}$ is still reasonably large.

Of course one of the main points of the whole construction is that it can be done without disturbing $\mathcal{C}_{\mu}(h)$ too much at each stage, so that at the end of the construction we still get that $\mathcal{C}_{\tilde{\mu}}(\tilde{h}) \in \operatorname{BMO}(\tilde{K}, d \tilde{\mu})$. Then one can apply Theorem 5.6 to $\mathcal{C}_{\tilde{\mu}}$ as above, and then conclude as before that most of $\tilde{K}$ (for $d \tilde{\mu}$ ) is rectifiable, and then a big piece of $K$ as well. The proof is reasonably easy to read; see $[\mathbf{C h 2}]$. Note that M. Christ was only able to get up to the $L^{2}$-boundedness of the Cauchy operator, because the Menger curvature trick did not exist at that time.

Now we have to say how to get rid of the assumption that $K$ is regular. Actually what really mattered in the construction of $[\mathbf{C h 2}]$ was the assumption that $\mu$ be doubling. This was shown by Y. Lin $[\mathbf{L i}]$.

For the last generalization, there are two slightly different approaches. The first one (in [DaMa], [Da5]) consists in keeping as close as possible to the proof above, and showing that each time we get in trouble because of bad doubling constants, we can compensate because this means that 
the density of our measure $\mu$ is getting smaller very fast when radii get smaller. Probably the main step is the construction of analogues on $K$ of dyadic cubes, with "small boundary properties" that allow a good coding of the compensation phenomenon above (when $\mu$ is far from doubling, its density decreases fast). The details are unfortunately painful, and it is difficult to give a good description here.

The approach in [NTV2] is slightly different. Instead of trying to construct "dyadic cubes" adapted to the geometry of $\mu$, they decide to use the standard dyadic cubes of the plane. Normally this should lead to disasters, because many of the estimates get bad when $\mu$ has lots of mass near the boundaries of cubes. Nazarov, Treil, and Volberg get away with this, by averaging the estimates over all dyadic grids parallel to the standard one, and a rather clever probabilistic argument. The result is that estimates are simpler, and also the statement of the generalization of Theorem 5.6 in [NTV1] is much nicer than the one in [Da5], because it does not rely on the "dyadic cubes" for the definition of BMO.

\section{Why doubling measures?}

To prove (9.1), we were forced in $[\mathbf{D a M a}]$ and $[\mathbf{D a 5}]$ to extend Calderón-Zygmund techniques to situations where the measure $\mu$ is not doubling. In [NTV1], the idea of getting rid of the usual condition of spaces of homogeneous type was more part of the initial motivation. At any rate, we now have at our disposal most of the usual boundedness results on singular integrals, in situations where we do not require a doubling condition on the ambient measure (but only upper bounds like (7.2)). The proofs are not even so much worse. See [NTV1] and [NTV3].

Thus for $L^{p}$-boundedness of singular integral operators, spaces of homogeneous type and doubling measures were not as natural as we have thought for a long time. Note that we may have been guilty of a similar oversight when studying uniform rectifiability (as in [DaSe]); maybe the systematic restriction to Ahlfors-regular sets (and in particular the lower bound in (6.2)) was sometimes superfluous. [It is certainly very convenient, though.]

\section{Boundedness of $\mathcal{C}_{\mu}$}

This is again a digression from our main theme (the Painlevé conjecture), but it is hard to resist mentioning that now, we know that for a finite Borel measure $\mu$ without atoms, 
(11.1) $\mathcal{C}_{\mu}$ is bounded on $L^{2}(d \mu)$ if and only if $\mu$ has linear growth (as in (7.2)) and satisfies the curvature condition (7.6).

As usual, a rigorous statement should address the issue of truncature. See [To1] for this and other similar results.

The first proof of (11.1) (by very little) is due to X. Tolsa [To1]; the proof used standard Calderón-Zygmund techniques, the "curvature operator" associated to integrals like in (7.4) and (7.6), the magic formula (7.1), and even some analytic capacity. The second proof is by Nazarov, Treil, Volberg [NTV1], as a direct consequence of their $T(b)$ theorem. There is now a direct proof by J. Verdera [Ve2], which is in the same spirit as Tolsa's, but simpler, and in particular does not use analytic capacity (but only Menger curvature).

\section{Conclusions}

Hopefully the reader is now convinced that now was about the time when (9.1) was to be proved. Of course it is always difficult to swear that all the technology that has been used was really needed. It seems however that Menger curvature and the magic formula (7.1) were really crucial in the argument (and indeed we do not know how to generalize the results in this text to higher dimensions). It is also pleasant to think that our conception of singular integral operators will benefit from the Vitushkin story (as in [NTV1], [NTV3] and Section 10).

The main question left is the characterization of vanishing analytic capacity for compact sets $K$ of dimension 1 but with $H^{1}(K)=+\infty$. Apparently we do not even know that unrectifiability is the answer when $H^{1}(K)$ is sigma-finite. The main problem is that when $H^{1}(K)=+\infty$ and $f \in H^{\infty}(\mathbb{C} \backslash K), f$ is not always the Cauchy integral of some measure, and then we don't know how to construct other bounded (or almost bounded) functions that are Cauchy integrals of measures. We do not know this even in the apparently simple case of Cantor sets like the Garnett-Ivanov example in Section 3, but with dilation ratios different from $\frac{1}{4}$ but tending to $\frac{1}{4}$. See [Ma3] for positive results.

M. Melnikov likes to conjecture that in all cases $\gamma(K)>0$ if and only if $K$ supports a nontrivial positive measure with linear growth (as in (7.2)) and finite Menger curvature $c^{2}(\mu)$ (see the definition (8.7)). The "if" part follows from $[\mathbf{M e l}]$, so only the converse is an issue. Apparently it follows from the proof of (9.1) in [NTV2] that if there is a finite complex measure $\nu$ such that the convolution $\frac{1}{z} * \nu$ is bounded, then there is a measure $\mu$ as needed. But we don't know much about the gap between $H^{\infty}(\mathbb{C} \backslash K)$ and bounded Cauchy integrals of (finite complex) measures. 
There are also questions about geometric properties of sets which support a non-trivial measure with linear growth and finite Menger curvature. For the sake of the discussion, let us call these Melnikov sets. Does (3.1) imply that $K$ is not a Melnikov set? [The other direction is false [JoMö].] Are all nontrivial connected sets Melnikov sets? [Since they have positive analytic capacity by 2.5 , you have to say yes if you trust the Melnikov conjecture above.] It seems that P. Jones recently gave an affirmative answer. Is it true that images of Melnikov sets by bilipschitz mappings of the plane are Melnikov sets? [The very special case of $C^{1+\epsilon}$ diffeomorphisms is treated in [To3].] A similar question can be asked about sets of positive analytic capacity, but in this case even the simpler question of invariance under the mapping $x+i y \rightarrow x+2 i y$ is not answered yet.

As was mentioned earlier, suitable analogues of Menger curvature and the magic formula are cruelly missing in higher dimensions [see [Far]], and the analogues of Theorem 7.5 (and even more (9.1)) are not known (but fairly likely). See $[\mathbf{H u}]$ for partial results in this direction.

\section{References}

[Ah] L. Ahlfors, Bounded analytic functions, Duke Math. J. 14 (1947), 1-11.

[Ca] A. P. Calderón, Cauchy integrals on Lipschitz curves and related operators, Proc. Nat. Acad. Sci. U.S.A. 74 (1977), 1324-1327.

[Ch1] M. Christ, "Lectures on singular integral operators," NSFCBMS Regional Conference series in Mathematics 77, AMS, 1990.

[Ch2] M. Christ, A $T(b)$ theorem with remarks on analytic capacity and the Cauchy integral, Colloq. Math. 60/61 (1990), 1367-1381.

[CJS] R. R. Coifman, P. Jones and S. Semmes, Two elementary proofs of the $L^{2}$-boundedness of Cauchy integrals on Lipschitz curves, J. Amer. Math. Soc. 2(3) (1989), 553-564.

[CMM] R. R. Coifman, A. McIntosh and Y. Meyer, L'intégrale de Cauchy définit un opérateur borné sur $L^{2}$ pour les courbes lipschitziennes, Ann. of Math. 116 (1982), 361-387.

[CoMe] R. R. CoIfman And Y. Meyer, "Wavelets, Calderón-Zygmund and multilinear operators," Cambridge Studies in Advanced Mathematics 48, Cambridge Univ. Press, 1997.

[CoWe] R. R. CoIfman AND G. Weiss, "Analyse harmonique noncommutative sur certains espaces homogènes," Lecture Notes in Math. 242, Springer-Verlag, 1971. 
[Da1] G. DAVID, Opérateurs intégraux singuliers sur certaines courbes du plan complexe, Ann. Sci. École Norm. Sup. 17 (1984), 157-189.

[Da2] G. DAVID, Morceaux de graphes lipschitziens et intégrales singulières sur une surface, Rev. Mat. Iberoamericana 4 (1988), 73-114.

[Da3] G. DAVID, "Wavelets and singular integrals on curves and surfaces," Lecture Notes in Math. 1465, Springer-Verlag, 1991.

[Da4] G. DAVID, Analytic capacity, Cauchy kernel, Menger curvature, and rectifiability, in "Harmonic Analysis and Partial Differential Equations," Proceedings of the symposium in honor of A. P. Calderón's birthday, Chicago, Feb. 1996. University of Chicago Press. To be published.

[Da5] G. DAVID, Unrectifiable 1-sets have vanishing analytic capacity, Rev. Mat. Iberoamericana 14(2) (1998), 369-479.

[DJS] G. David, J. L. Journé and S. Semmes, Opérateurs de Calderón-Zygmund, fonctions para-accrétives et interpolation, Rev. Mat. Iberoamericana 4 (1985), 1-56.

[DaMa] G. David And P. Mattila, Removable sets for Lipschitz harmonic functions in the plane, Rev. Mat. Iberoamericana. To be published.

[DaSe] G. DAVID AND S. SEMmes, "Analysis of and on uniformly rectifiable sets," AMS series of Mathematical surveys and monographs 38, 1993.

[Dv] A. M. DAvie, Analytic capacity and approximation problems, Trans. Amer. Math. Soc. 171 (1972), 409-444.

[DØK] A. M. DAVIE AND B. ØKSEndAL, Differentiability properties of finely harmonic functions, Acta Math. 149 (1982), 127-152.

[Fal] K. FALCONER, "The geometry of fractals sets," Cambridge Univ. Press, 1984.

[Far] H. M. FARAG, The Riesz kernels do not give rise to higher dimensional analogues of the Menger-Melnikov curvature, Publ. Mat. 43 (1999), 251-260.

[Fe] H. FEDERER, "Geometric measure theory," Grundlehren der mathematischen Wissenschaften 153, Springer-Verlag, 1969.

[Ga1] J. Garnett, Positive length but zero analytic capacity, Proc. Amer. Math. Soc. 21 (1970), 696-699.

[Ga2] J. GarnetT, "Analytic capacity and measure," Springer-Verlag, 1972.

[HaHa] V. P. Havin And D. Havinson, see the references 15 on page 489 and 7, 8 on page 493, in "Linear and Complex Analysis 
Problem Book," (V. P. Havin, S. V. Hruscëv and N. K. Nikolskii, eds.), Lecture Notes in Mathematics 1043, Springer-Verlag, 1984.

[Hu] P. HuOvinen, Singular integrals and rectifiability of measures in the plane, Ann. Acad. Sci. Fenn. Ser. A I Math. Dissertationes 109 (1997), 63 pp.

[Iv] L. D. Ivanov, "Variations of sets and functions," Nauka i Tekhn. Progress, 1975 (in russian).

[Jo1] P. Jones, Square functions, Cauchy integrals, analytic capacity, and harmonic measure, in "Proc. Conf. on Harmonic Analysis and Partial Differential Equations," El Escorial 1987 (J. García-Cuerva, ed.), Lecture Notes in Math. 1384, Springer-Verlag, 1989, pp. 24-68.

[Jo2] P. Jones, Rectifiable sets and the traveling salesman problem, Invent. Math. 102 (1990), 1-16.

[JoMu] P. Jones And T. Murai, Positive analytic capacity but zero Buffon needle probability, Pacific J. Math. 133 (1988), 99-114.

[JoMö] H. Joyce AND P. MÖrTERs, A set with finite curvature and projections of zero length, Preprint (1997).

[Lé] J.-C. LÉGER, Menger curvature and rectifiability, Ann. of Math. (to appear).

[Li] Y. Lin, Menger curvature, singular integrals and analytic capacity, Ann. Acad. Sci. Fenn. Ser. A I Math. Dissertationes 111 (1997), $44 \mathrm{pp}$.

[MaSe1] R. A. Macias AND C. Segovia, Lipschitz functions on spaces of homogeneous type, Adv. Math. 33 (1979), 257-270.

[MaSe2] R. A. Macias And C. Segovia, A decomposition into atoms of distributions on spaces of homogeneous type, Adv. Math. 33 (1979), 271-309.

[Mar] D. E. MARshall, Removable sets for bounded analytic functions, in "Linear and Complex Analysis Problem Book," (V. P. Havin, S. V. Hruscëv and N. K. Nikolskii, eds.), Lecture Notes in Mathematics 1043, Springer-Verlag, 1984, pp. 485-490.

[Ma1] P. MatTilA, Smooth maps, null-sets for integral geometric measure and analytic capacity, Ann. of Math. 123 (1986), 303-309.

[Ma2] P. MattilA, "Geometry of sets and measures in Euclidean space," Cambridge Studies in Advanced Mathematics 44, Cambridge Univ. Press, 1995.

[Ma3] P. MatTila, On the analytic capacity and curvature of some Cantor sets with non- $\sigma$-finite length, Publ. Mat. 40 (1996), 127-136.

[Ma4] P. Mattila, Removable sets for bounded analytic functions, Proc. of the Conference in honor of Matts Essen, Uppsala 1997. To 
be published.

[Ma5] P. Mattila, Rectifiability, analytic capacity, and singular integrals, Proc. of the ICM, Vol. II (Berlin, 1998), Doc. Math. Extra Vol. II (1998), 657-664 (electronic).

[MMV] P. Mattila, M. Melnikov And J. Verdera, The Cauchy integral, analytic capacity, and uniform rectifiability, Ann. of Math. 144 (1996), 127-136.

[Mel] M. Melnikov, Analytic capacity: discrete approach and curvature of measure, Russian Acad. Sci. Sb. Math. 186 (1995), 827-846.

[MeVe] M. Melnikov And J. VerderA, A geometric proof of the $L^{2}$ boundedness of the Cauchy integral on Lipschitz graphs, Internat. Math. Res. Notices 7 (1995), 325-331.

[Mey] Y. MeYER, "Ondelettes et Opérateurs II: Opérateurs de Calderón-Zygmund," Actualités Mathématiques, Herman, Paris, 1990.

[Mu1] T. MurAi, Comparison between analytic capacity and the Buffon needle probability, Trans. Amer. Math. Soc. 304 (1987), 501514.

[Mu2] T. MurAI, "A real variable method for the Cauchy transform and analytic capacity," Lecture Notes in Math. 1307, SpringerVerlag, 1988.

[NTV1] F. Nazarov, S. Treil and A. Volberg, Cauchy integral and Calderón-Zygmund operators on non homogeneous spaces, Internat. Math. Res. Notices 15 (1997), 703-726.

[NTV2] F. Nazarov, S. Treil AND A. VolberG, Pulling ourselves by the hair (the proof of the Vitushkin conjecture), Preprint Michigan State Univ., available at home pages at http://www.math.msu.edu/.

[NTV3] F. Nazarov, S. Treil and A. VolberG, Weak type estimates and Cotlar inequalities for Calderón-Zygmund operators on non homogeneous spaces, Internat. Math. Res. Notices 9 (1998), 463-487.

[Pa1] H. PAJot, Sous-ensembles de courbes Ahlfors-régulières et nombres de Jones, Publ. Mat. 40 (1996), 497-526.

[Pa2] H. PAJot, Conditions quantitatives de rectifiabilité, Bull. Soc. Math. France 125 (1997), 15-53.

[St] E. M. STEIn, "Harmonic Analysis: real-variable methods, orthogonality, and oscillatory integrals," Princeton Univ. Press, 1993.

[To1] X. TolsA, $L^{2}$-boundedness of the Cauchy integral operator for continuous measures, Duke Math. J., to be published.

[To2] X. TolsA, Cotlar's inequality and existence of principal values 
for the Cauchy integral without doubling condition, J. Reine Angew. Math., to be published.

[To3] X. TolsA, Curvature of measures, Cauchy singular integral and analytic capacity, Thesis, Universitat Autònoma de Barcelona (1998).

[Uy1] N. X. Uy, Removable sets of analytic functions satisfying a Lipschitz condition, Ark. Mat. 17 (1979), 19-27.

[Uy2] N. X. Uy, A removable set for Lipschitz harmonic functions, Michigan Math. J. 37 (1990), 45-51.

[Ve1] J. VERDERA, Removability, capacity and approximation, in "Complex Potential Theory," NATO ASI Series, Kluwer Academic Publ., Dordrecht, 1994, pp. 419-473.

[Ve2] J. VERDERA, On the T(1)-Theorem for the Cauchy integral, Prepublicacions Universitat Autònoma de Barcelona 16/1998, Ark. Mat. (to appear).

[Vi1] A. G. Vitushkin, Example of a set of positive length but zero analytic capacity, Dokl. Akad. Nauk SSSR 127 (1959), 246-249 (in russian).

[Vi2] A. G. Vitushkin, The analytic capacity of sets in problems of approximation theory, Uspekhi Mat. Nauk 22 (1967), 141-199 (english trans.); Math. Surveys Monographs 22 (1967), 139-200 (in russian).

[VIM] A. G. Vitushinin, L. D. Ivanov And M. Melnikov, Incommensurability of minimal linear measure with the length of a set, Dokl. Akad. Nauk 151 (1963), 1256-1259 (in russian); Soviet Mathematics 4 (1963), 1160-1164 (in English).

\author{
Department of Mathematics \\ Université Paris-Sud \\ 91405 Orsay Cedex \\ FRANCE \\ e-mail: Guy.David@math.u-psud.fr
}

Rebut el 2 de novembre de 1998 